Sarcomatoid carcinoma (SC) is a rare primary malignant tumor in which both carcinomatous and sarcomatous elements occur. It can occur in many different organs and anatomical locations, such as the skin, thyroid gland, bone, urinary tract, breast, pancreas, liver and other areas. Of them, pulmonary sarcomatoid carcinoma (PSC) is a rare malignant cancer composed of sarcoma and sarcoma-like tumors with spindle or giant cell features. Here a case of a 75-yearold Chinese man with a six-month history of cough and hemoptysis is reported. Chest X-ray showed a tumor shadow in the left lung field. Chest computed tomography (CT) scan showed a lobulated mass in his left hilum and even the left pulmonary artery. Pleomorphic interstitial cells were found by bronchoscopic brushing. To establish a definitive diagnosis for PSC, a left pneumonectomy was performed. The pathological stage was IIB (pT2N1M0) based on the tumor node metastasis (TNM) staging system. The tumor's pathology, histology, immunohistochemistry and treatment methods are discussed.

Key words: lung neoplasms; carcinoma; diagnosis; treatment.

\section{Pulmonary sarcomatoid carcinoma: a case report}

\author{
Xiao-yong Shen ${ }^{1 *}$, Zhi-feng Lin ${ }^{2 \star}$, Qiang Lin $^{2}$, Zhen Ruan², Hai-long Huang ${ }^{2}$, \\ Chao-qiang Ju', Jin Wang ${ }^{2}$
}

1Department of Thoracic Surgery, Huadong Hospital, Shanghai Fudan University, Shanghai, China

2Department of Thoracic Surgery, Shanghai First People's Hospital, Shanghai Jiaotong University, Shanghai, China

*The authors wish it to be known that in their opinion, the first two authors should be regarded as joint first authors.

\section{Introduction}

Sarcomatoid carcinoma (SC) is a rare primary malignant tumor in which both carcinomatous and sarcomatous elements occur. It can occur in many different organs and anatomical locations, such as the skin, thyroid gland, bone, urinary tract, breast, pancreas, liver and other areas [1-6]. Of them, pulmonary sarcomatoid carcinoma (PSC) is particularly uncommon, representing less than $1 \%$ of all lung tumors [7]. We herein report a PSC case to discuss the clinical manifestations, diagnosis, treatment, and prognosis of this condition.

\section{Case report}

On March 10, 2011, a 75-year-old man was admitted to our department because of coughing and hemoptysis for six months. He also had a 45-year history of cigarette smoking (20 cigarettes per day) and a significant medical history of hypertension. His chest $\mathrm{X}$-rays showed a tumor shadow presenting in the left lung field (arrow in Fig. 1). Chest computed tomography (CT) scan revealed a lobulated mass occupying the left hilum and even stretching mediastinal structures including the left pulmonary artery (arrow in Fig. 2). Bronchoscopic examination demonstrated significant obstruction involving the superior segmental bronchus (BVI) due to pressure by abnormal vessels. Pleomorphic interstitial cells were further observed by bronchoscopic brushing.

However, for definitive diagnosis of this disease, a left pneumonectomy and lymph node dissection were performed on March 18, 2011. The results showed a $6.5 \times 6.5 \times 6 \mathrm{~cm}^{3}$ lobulated mass in the left hilum. The tumor was ovoid in shape, poorly circumscribed, yellow-tan in color, heavy, hemorrhagic, and necrotic. Histologically, the tumor was composed of compact cells arranged in cords. The tumor cells were pleomorphic, irregular, ovoid, or spindle-like in shape, and some of them had an indistinct cell boundary. Importantly, severe necrosis was found inside (Fig. 3). Immunohistochemically, the tumor cells were positive for epithelial membrane antigen (EMA) (Fig. 4A), creatine kinase (CK) (Fig. 4B), and vimentin (Fig. 4C). They were negative for thyroid transcription factor-1 (TTF-1) (Fig. 4D), smooth muscle actin (SMA) (Fig. 4E), and cytokeratin 5/6 (Fig. 4F). Based on the new revision of the tumor node metastasis (TNM) staging system, this pulmonary carcinoma was classified as stage IIB (pT2N1MO).

\section{Discussion}

Pulmonary sarcomatoid carcinomas (PSCS) are poorly differentiated nonsmall cell lung carcinomas (NSCLCS) containing a sarcoma-like element (malignant spindle or giant cells) or sarcomatous component (neoplastic bone, cartilage, or striated muscle) [8]. In the World Health Organization (WHO) clas- 


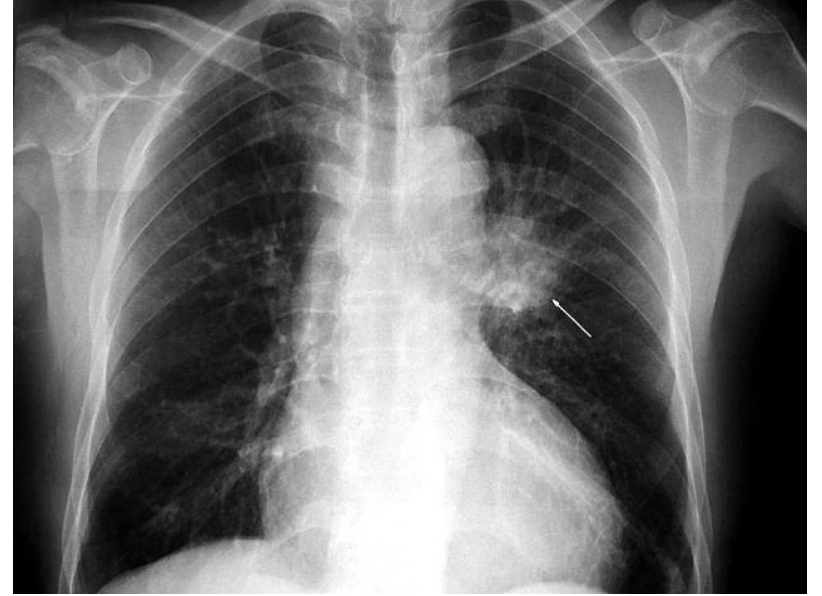

Fig. 1. Tumor shadow in the left lung

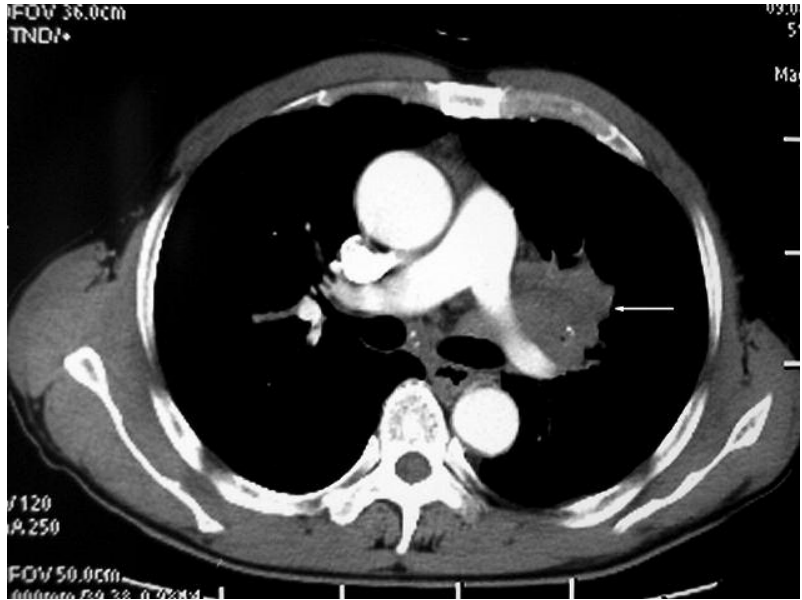

Fig. 2. Lobulated mass in the left hilum and even the left pulmonary artery
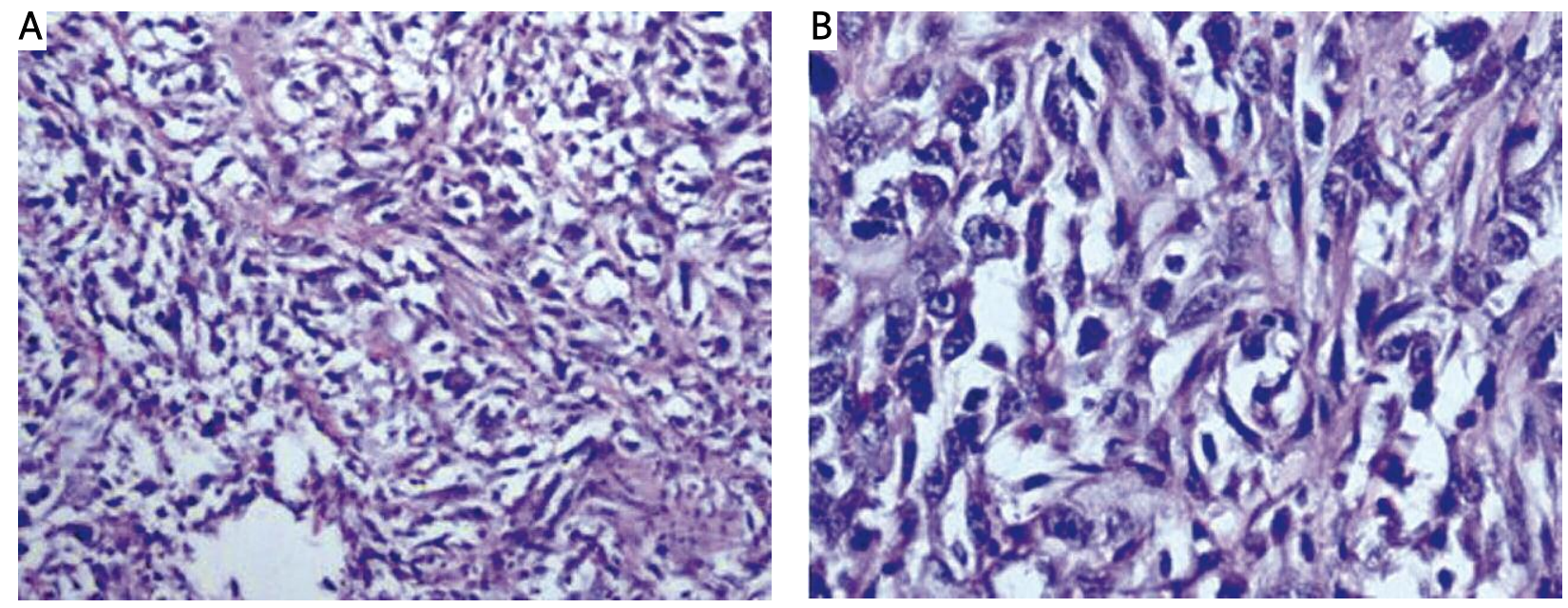

Fig. 3. Histological analysis of the resected tumor specimen A) Tumor cells were pleomorphic, irregular, ovoid, or spindle-like in shape, and some of them had an indistinct cell boundary. The tumor was composed of compact cells arranged in cords (HE, magnification 200x). B) A higher magnification of the sarcomatoid component (HE, magnification 400x)

sification, spindle cell carcinoma is classified as a variant of squamous cell carcinoma and giant cell carcinoma is classified as a variant of large cell carcinoma [9]. However, Fishback et al. designated SC as pleomorphic (spindle/giant cell) carcinomas of the lung because both spindle and giant cell components are often found in the same tumors [10].

PSC patients are predominantly male and smokers, with a male-to-female ratio of $4.4: 1$ and average age at presentation of 51.4 years in the published series [11]. The patient in our case also had a long history of cigarette smoking. Besides, it is believed by some authors that radiotherapy for the treatment of a patient's other cancers may also induce a sarcomatoid change in lung tissue, which can accelerate the subsequent development of SC [12].

Pulmonary sarcomatoid carcinoma presents as either a central or peripheral lesion, most often in the right upper lobes. It grows by invading the bronchial tree, the pulmonary parenchyma, and the adjacent anatomical structures (mediastinum and chest wall) in the form of widely necrotic and hemorrhagic, round to bosselated large masses [13]. In our case, the lobulated mass occupied the left hilum and even stretched to mediastinal structures, such as the left pulmonary artery. Significant BVI obstruction was also observed. The tumor was ovoid in shape, poorly circumscribed, yellow-tan in color, heavy, hemorrhagic, and necrotic.

The clinical signs and symptoms may be related to tumor localization. Symptoms such as chest pain, accumulation of excessive fluid in the pleural cavity, and difficulty breathing are shared by most pulmonary carcinomas. This makes it hard to distinguish them from each other. In endobronchial tumors, coughing and blood-tinged sputum usually occur, but peripheral tumors are asymptomatic [14]. However, no paraneoplastic syndrome was reported to accompany PSC, although such symptoms occurred in about $15-20 \%$ of small cell lung carcinomas (SCLCS) and 5-8\% of NSCLCs [15]. PSCs metastasize via lymph and blood vessel routes to the same anatomical sites as conventional NSCLCs. Therefore, in general, PSC is classified using the same TNM criteria as other NSCLCS.

Although the final diagnosis of PSC depends on the close scrutiny of histopathology, its immunohistochemical analysis may help to better highlight the different cell components 

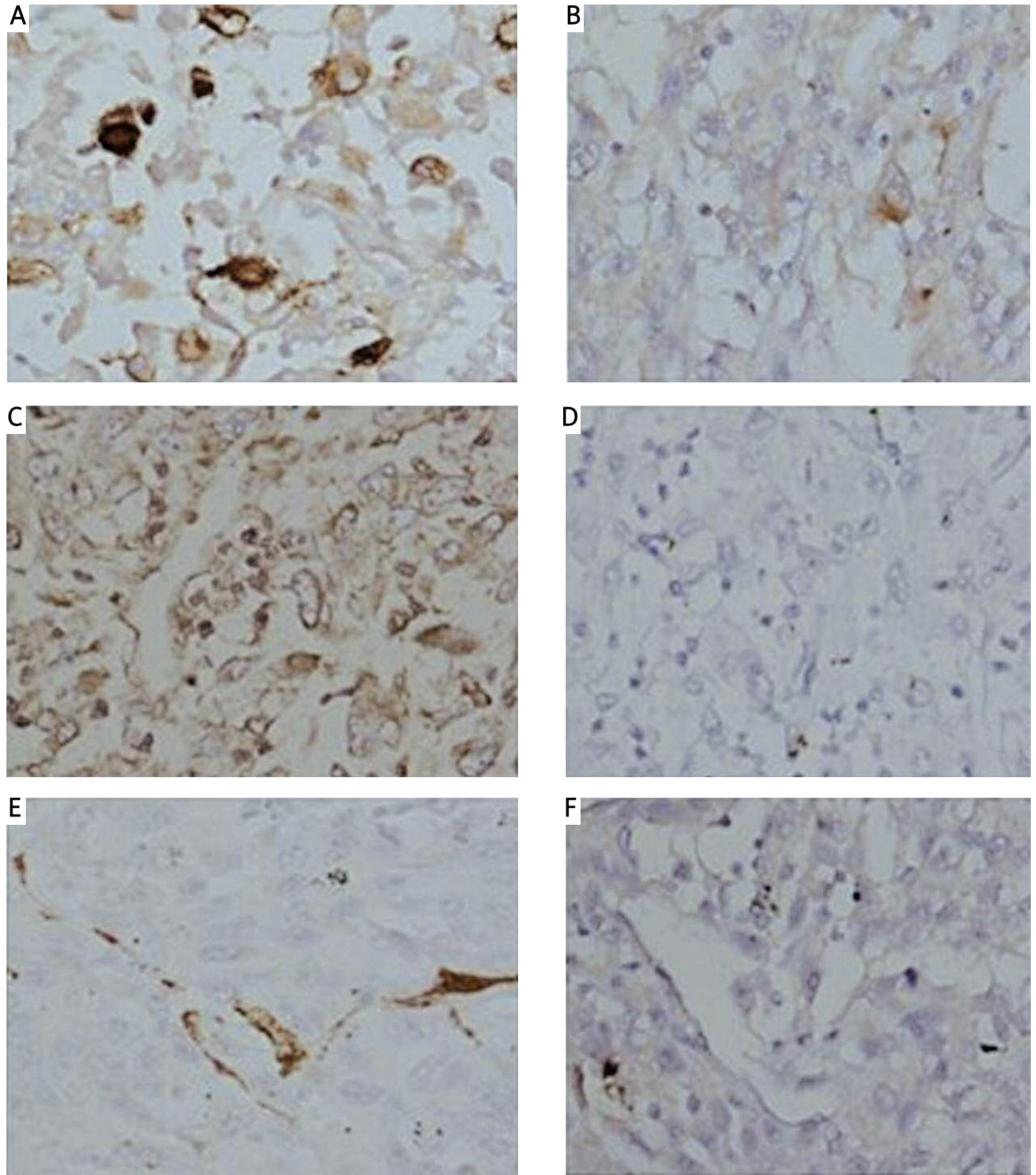

Fig. 4. Immunohistochemical staining of the resected tumor specimen. There was positive expression of: A) EMA, B) CK, and C) vimentin, but negative expression of D) TTF-1, E) SMA, and F) cytokeratin 5/6

occurring in these tumors [16]. The epithelial component is stained with antibodies to cytokeratins, EMA, and carcinoembryonic antigen (CEA), whereas the opposite holds true for the sarcomatous or sarcomatoid component, which is instead consistently immunoreactive for vimentin. In our case, we found that the PSC specimen was positive for EMA, CK, and vimentin, but negative for TTF-1, SMA, and cytokeratin $5 / 6$. This result suggested that our case had sarcomatoid components and epithelial phenotype, which was in accordance with previous reports [17-19].
Pulmonary sarcomatoid carcinoma is an aggressive cancer and the resultant tumors are frequently symptomatic, locally advanced, and have high rates of recurrence. Recently, although radical surgery is still reported as the best option for PSC patients [20], many non-surgical treatments are recommended for patients with clinically advanced sarcomatoid cancer, such as chemotherapy. Chemotherapy is often performed to treat patients according to the results of trials that enrolled patients with lung squamous cell carcinoma or adenocarcinoma. Italiano et al. [21] reported the short- 
term outcomes of using doxorubicin and ifosfamide in the treatment of metastatic SC. Half of their patients with metastatic SC experienced an initial response to chemotherapy with doxorubicin and ifosfamide. However, all patients ultimately died of disease despite continued treatment [22]. Therefore, due to the limited treatment, the five-year survival rate for patients with PSC was only $24.5 \%$, relatively worse than for patients with other forms of NSCLC (46.3\%).

The authors declare no conflicts of interest. We wish to express our warm and sincere thanks to Weidong Zang and Lishan Wang from Shanghai Jiaotong University and Fenghe Information and Technology Inc. Their ideas and help brought a remarkable improvement in our research.

\section{References}

1. Inaloz H, Ayyalaraju R, Holt P, Laidler P. A case of sarcomatoid carcinoma of the skin. J Eur Acad Dermatol Venereol 2003; 17: 59-61.

2. Okoń K, Wierzchowski W, Jabłońska E, Wójcik P, Steczko A. Anaplastic, sarcomatoid carcinoma of the thyroid originating from a Hürthle cell tumor. Pol J Pathol 2003; 54: 277-81.

3. Ikegami H, Iwasaki H, Ohjimi Y, Takeuchi T, Ariyoshi A, Kikuchi M. Sarcomatoid carcinoma of the urinary bladder: A clinicopathologic and immunohistochemical analysis of 14 patients. Hum Pathol 2000; 31: 332-40.

4. Terada T. Urinary bladder carcinoma with triplicate differentiations into giant cell sarcomatoid carcinoma, squamous cell carcinoma, and papillary urothelial transitional cell carcinoma: a case report. Cases J 2009; 2: 9111.

5. Haruki T, Maeta H, Sawazumi Y, Miyasaka S, Morimoto K, Ishikawa N, Nakamoto S, Taniguchi I. Biphasic metaplastic sarcomatoid carcinoma of the breast: report of a case. Breast Cancer 2009; 16: 229-233.

6. De la Riva S, Muńoz-Navas MA, Betés M, Súbtil JC, Carretero C Sola JJ. Sarcomatoid carcinoma of the pancreas and congenital choledochal cyst. Gastrointest Endosc 2006; 64: 1005-6.

7. Travis WD. Pathology and genetics of tumours of the lung, pleura, thymus and heart. larc 2004.

8. Franks TJ, Galvin JR. Sarcomatoid carcinoma of the lung: histologic criteria and common lesions in the differential diagnosis. Arch Pathol Lab Med 2010; 134: 49-54.

9. Beasley MB, Brambilla E, Travis WD. The 2004 World Health Organization classification of lung tumors. In Edition Elsevier 2005; 90-97.

10. Fishback NF, Travis WD, Moran CA, Guinee DG Jr, McCarthy WF, Koss MN. Pleomorphic (spindle/giant cell) carcinoma of the lung. A clinicopathologic correlation of 78 cases. Cancer 1994; 73: 2936-2945.

11. DH D, SP H. Cancer Sci 1987; 98: 1914-1920.

12. Nakajima M, Kasai T, Hashimoto H, Iwata Y, Manabe H. Sarcomatoid carcinoma of the lung. Cancer 1999; 86: 608-616.

13. Pelosi G, Sonzogni A, De Pas T et al. Review article: pulmonary sarcomatoid carcinomas: a practical overview. Int J Surg Pathol 2010; 18: 103-20.

14. Sanyal K, Sabanathan K. Lung carcinosarcoma as a rare biphasic sarcomatoid carcinoma: a case report. Cases J 2009; 2: 7968.

15. Hansen M. Paraneoplastic syndromes and tumor markers for small cell and non-small cell lung cancer. Curr Opin Oncol 1990; 2: 345-51.

16. Lewis JS, Ritter JH, El-Mofty S. Alternative epithelial markers in sarcomatoid carcinomas of the head and neck, lung, and bladderp63, MOC-31, and TTF-1. Mod Pathol 2005; 18: 1471-81.

17. Terada T. Sarcomatoid carcinoma of the lung presenting as a cutaneous metastasis. J Cutan Pathol 2010; 37: 482-5.

18. Jiang M, Cao D, Yang Y, Gou H. Clinical analysis of sarcomatoid carcinoma of the lung. Zhongguo Fei Ai Za Zhi 2006; 9: 547-9.

19. Hountis P, Moraitis S, Dedeilias P, Ikonomidis P, Douzinas M. Sar comatoid lung carcinomas: a case series. Cases J 2009; 2: 7900

20. Petrov DB, Vlassov VI, Kalaydjiev GT, Plochev MA, Obretenov ED, Stanoev VI, Danon SE. Primary pulmonary sarcomas and carcinosar- comas-postoperative results and comparative survival analysis. Eur J Cardiothorac Surg 2003; 23: 461-6..

21. Italiano A, Cortot AB, Ilie M, et al. EGFR and KRAS status of primary sarcomatoid carcinomas of the lung: Implications for anti-EGFR treatment of a rare lung malignancy. Int J Cancer 2009; 125: 2479-82.

22. Akao Y, Nakagawa Y, Kitade Y, Kinoshita T, Naoe T. Downregulation of microRNAs-143 and -145 in B-cell malignancies. Cancer Sci 2007; 98: 1914-20.

\section{Address for correspondence}

\section{Qiang Lin}

Department of Thoracic Surgery

Shanghai First People's Hospital

Shanghai Jiaotong University

Shanghai, China

tel. +86-021-63240090

e-mail: xklinqiang@hotmail.com

Submitted: 15.05 .2012

Accepted: 24.09.2012 